\title{
SNe FROM SURVEYS AND PATROLS
}

\author{
C. POLLAS \\ Télescope de Schmidt \\ Observatoire de la Côte d'Azur \\ Caussols \\ F-06460 St Vallier de Thiey, France
}

\section{SNe Discoveries after Zwicky}

The name of Zwicky is attached to the first photographic Palomar Survey and to the History of SNe study. An exciting study resumed by the striking image $(M=-19)$ of a transient source appearing at extragalactic distance, as bright as the total magnitude of its parent galaxy. Expected to explode once a century, such an event is rare. But the more galaxies are checked the more $\mathrm{SNe}$ are found. That is why the wide-field is a good tool. The Palomar team SNe activity was followed by a quiet period of the whole SNe discovery (Fig. 1); from those the Cero El Robles search emerged in the early eighties. Several important patrols continued with Schmidt telescopes of size about $0.5 \mathrm{~m}$ (Bern, Hungary, Urss...) in spite of the low level of SNe found. Important because knowing that no SN exploded in a galaxy (especially in the sample of nearby galaxies) is the basis of the statistics. The SNe production increased in the late eighties. At the same time, the closest modern SN (1987A) led for the first time to the use of a number of instruments

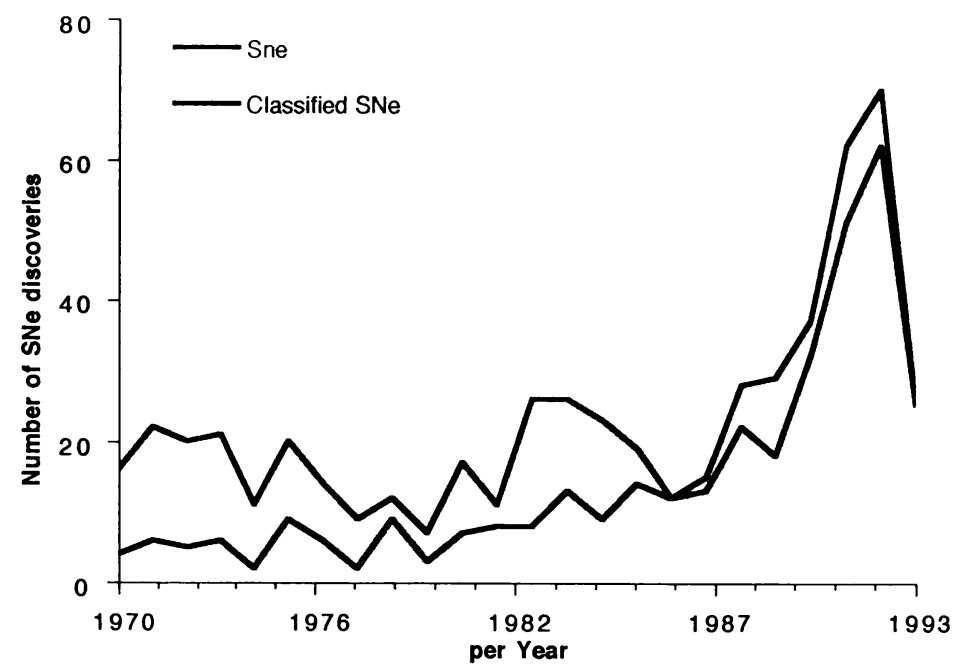

Figure 1. SNe discoveries EVOLUTION. 
complementing each other to increase our understanding of SNe. But more important than the production of new SNe, is the increase of their spectroscopic classification which is necessary to the $\mathrm{SNe}$ work.

A. Filippenko started his intensive follow-up in 1985 at the Lick Observatory. In this way, he encouraged the wide-field quest of $\mathrm{SNe}$ in the course of other astronomical programs on Schmidt telescopes (OCA, Palomar, Siding Spring) (see too the R. Kirshner's work with the MMT). The eighties were marked by two surveys with small field instruments. The first, still continuing, was a visual search by the amateur $R$. Evans who produced the basic data of the statistical analysis (van den Bergh \& Tamman 1991). The second was the Berkely Automated SNe search (Perlmutter et al. 1988) who perfected the systematic and objective checking of an extended number of nearby galaxies (up to the technical limits). This did not produce a lot of new SNe but pointed out a qualitative result (Muller et al. 1992) concerning the frequent explosion of massive stars deep inside galaxy images; hidden to the shorter focus community with wide-field telescopes. Today the automated survey continues at the Berkeley University in a successful new way (Treffers et al. 1993) associated with a spectroscopic and photometric followup. A few other projects are using CCDs like the Perth Observatory Group. Also, by associating spectroscopy and photometry with large field scans, the Calan/CTIO (Curtis Schmidt) SNe search is the more productive. Data used in the figures are provided by the IAU circulars (B.G. Marsden, D.W.E. Green) and the Asiago catalogue of SNe, up to date in 1993 (R. Barbon, E. Cappellaro, M. Turatto).

Here are summarised annual SN discovery rates (averaged):

\begin{tabular}{c|l|l|c|c|c|c}
\hline $\begin{array}{l}\text { Regular surveys } \\
\text { serendipitous } \\
\text { discoveries }\end{array}$ & $\begin{array}{l}\text { BASS, LOSS, PORG } \\
\text { automated +CCD }\end{array}$ & $\begin{array}{l}\text { R. } \\
\text { Evans }\end{array}$ & $\begin{array}{l}\text { OCA, POSS, UKST } \\
\text { large Schmidt }\end{array}$ & $\begin{array}{l}\text { Calan/CTIO } \\
\text { (Ce1R) }\end{array}$ \\
\hline \hline 8 & 3 & 2 & 30 & $(3 \times 10)$ & 20 & $(6)$ \\
\hline $1980-1992$ & $1986-$ & $1981-$ & $1988-$ & & $1991-$ & $\begin{array}{l}(1980 \\
1987)\end{array}$ \\
\hline
\end{tabular}

Figure 2 shows us the complementarity of the SNe discoveries. Only less than 5\% are jointdiscoveries. The wide-field patrols contribute to the faintest discoveries, in the huge sample of galaxies of magnitude $B=18$ which overlaps the current catalogues of galaxies.

\section{A Schmidt SNe Search at OCA}

The photographic SNe search at the OCA Schmidt telescope (90/150/316), a $5^{\circ} \times 5^{\circ}$ field up to the 22nd magnitude operated with A. Maury and D. Albanese, is conducted by me in a different way from the other large Schmidt, since I spent on average 7 hours per field where other researchers spent 30 mins. The OCA production is only 200 films or plates per year or 10 times less than those of Palomar or Siding Spring.

Some sixty SNe were discovered of which two thirds are classified: half in type Ia, the other in II or Ib/c. The sixty includes many subtypes and peculiarities. For $25 \mathrm{SNe}$, their age (from maximum) was estimated. For these, a third were discovered before or close to maximum, another one third, from 1 to 3 weeks after maximum and the last third 1 to several months past maximum. My monitoring provides about one SN per 3000 galaxies and about one SN per 5 


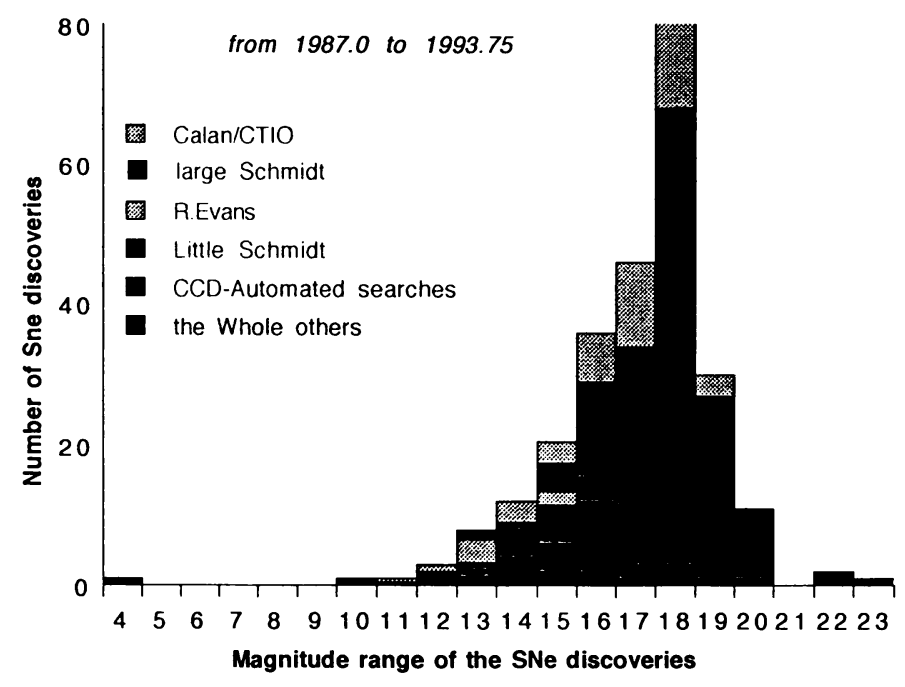

Figure 2. Who does what?

fields. Figure 3 shows some photographs of the appearance of such SNe down to the faintest found.

\section{Weakness and Interest of the Wide-Field SNe Patrols}

Among the well detected events are:

1) the absolutely bright such as type Ia $(M=-20)$;

2) SNe which are distant from the centre of the host galaxy;

3) $\mathrm{SNe}$ on intrinsically faint or diffuse galaxy image.

A quick analysis of the classified SNe shows that:

1) SNe in distant galaxies appear with larger offset;

2) type II Sne are more often found in spiral face-on galaxies (providing the furthest type II SNe discovered).

In the faintest SNe images, I expect to detect some distant SN in 20th magnitude galaxies of small distant clusters of galaxies. The low rate of observation for each galaxy is balanced by the high number of galaxies obtained with wide-fields. SNe at $z=0.1$ are important for the preparation of more ambitious projects (Large telescopes, Space telescope) looking for cosmological SNe.

But the differences between the small and wide-field searches indicate the effects of external and internal absorption, often hardly taken into account in elaborating a new search project (which are based on statistics from the survey of nearby galaxies). These projects need spectroscopic observations which are prepared by the observation of faint $\mathrm{SNe}$ discovered with wide-field 


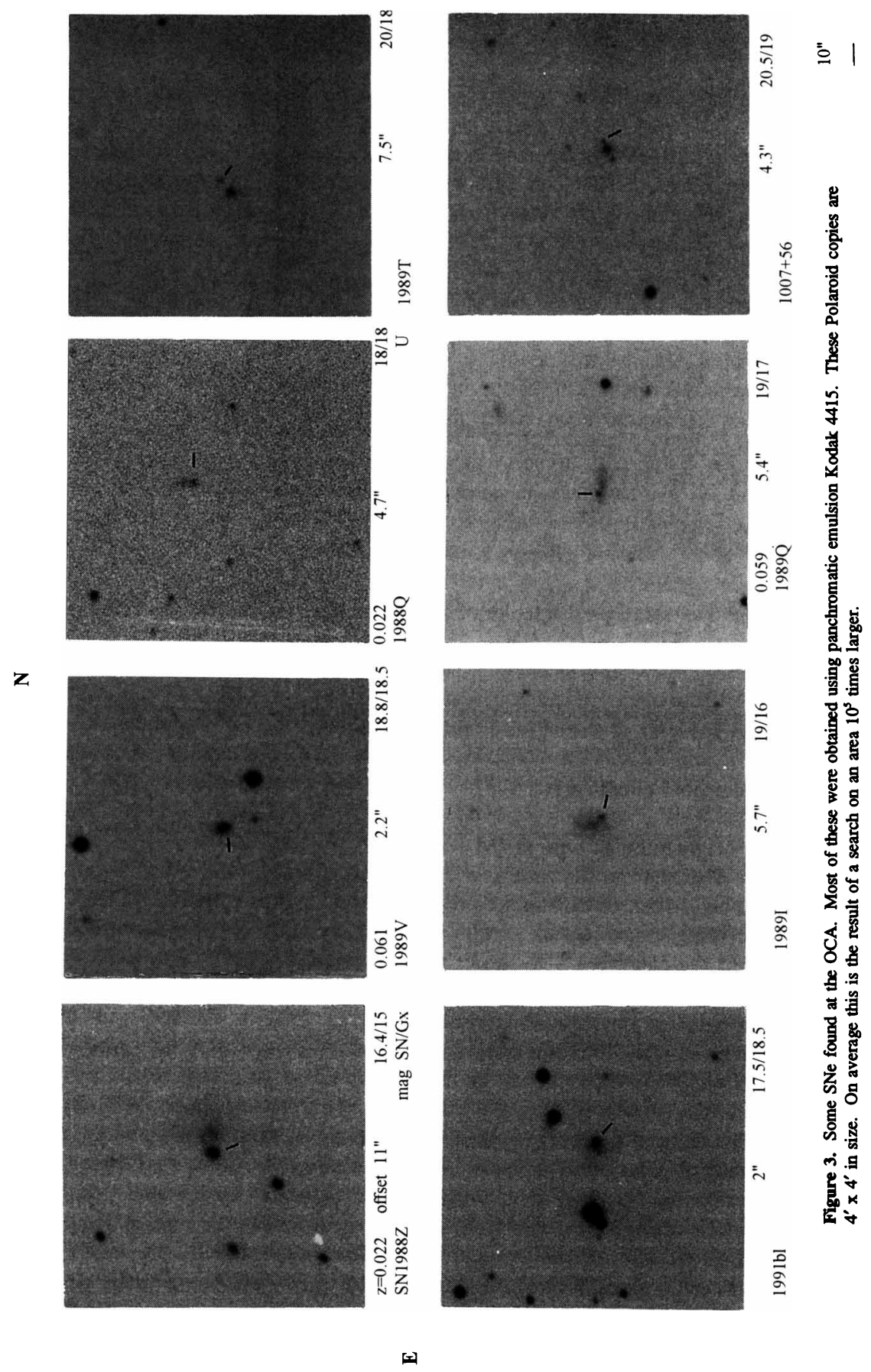


telescopes.

As pointed out during this Symposium, photographic wide-field surveys are also important as archival material. The progenitor of the SN 1993J showed how important is the record of widefields for the nearby SNe. In spite of their currently limited statistical use, the wide-field SN patrols give a good framework for future $\mathrm{SNe}$ explorations.

\section{References}

Muller, R.A. et al., 1992. Astrophys. J., 384, L9.

Perlmutter, S., et al., 1988. In 'Instrumentation for Ground-based Optical Astronomy', ed. L.B. Robinson, p. 674.

Treffers, R.R., et al., 1993. 'Leuschner Observatory Supernova Search', UC Berkeley. van den Bergh, S. and Tammann, G.A., 1991. Ann. Rev. Astron. \& Astrophys., 29, 363. 